Вісник Дніпропетровського університету. Серія: геологія, географія. 2016. 24 (1), 102-106.

Vìsnik Dnìpropetrovs'kogo unìversitetu. Seriâ geologîâ, geographîâ

Dnipropetrovsk University Bulletin. Series geology, geography. 2016, 24 (1), 102-106.

doi: $10.15421 / 111615$

http://geology-dnu.dp.ua

УДК 624.131 (477)

\title{
Опыт прогноза инженерно-геологических свойств просадочных горизонтов для целей проектирования сооружений методом нейронных сетей
}

\author{
Т. П. Мокрицкая, Д. О. Калинина \\ Днепропетровский национальный университет имени Олеся Гончара Днепропетровск, Украина, \\ e-mail: mtatjana@,bk.ru
}

Целью работы является применение индуктивного моделирования для прогноза изменений свойств грунтов. Охарактеризованы закономерности построения нейронных сетей. Выполнено моделирование зависимости показателей просадочности, деформируемости и прочности от пространственных координат (глубины) и показателей физического состояния. Выяснено, как полученные прогнозные значения будут влиять на расчеты оснований сооружений. Учитывая состояние жилого фонда, необходимость снижения инвестиционных рисков строительной деятельности, данная работа является чрезвычайно актуальной.

Ключевые слова: нейронная сеть, просадочность, деформируемость, основание сооружений

\section{Experience the forecast of engineering geological properties subsiding horizons for designing of neural network method}

\author{
T. P. Mokritskaya, D. O. Kalinina
}

Oles Honchar Dnipropetrovsk National University, Dnepropetrovsk, Ukraine, e-mail: mtatjana@,bk.ru

The object of study: Geological environment that is within the influence of the designed structures located on housing estate Topol-3, in the Dnepropetrovsk city. Objective: Justification optimal method forecast of engineering-geological processes on the results of the application of neural network technology. Method of research: quantitative and qualitative analysis of the conditions and factors for the development of engineering-geological processes in the zone of influence of facilities. Mathematical modeling, statistics, correlation and regression analyzes of neural network technology. For the first time in Ukraine had applied neural network technology to forecast changes in soil properties in the zone of influence of facilities that provide new opportunities for building design tasks. It was found that the areas of research in the geological structure of the array participate aeolian-diluvial quaternary sediments. In the geological section, explore to a depth of $26 \mathrm{~m}$, the array is allocated 6 geotechnical elements (GE), within which the thickness is statistically homogeneous in their properties. From the analysis of the conditions and factors of development of engineering-geological processes it is known that it may develop by settling and subsidence. Statistical homogeneity of the sample data on the properties of the soil allows you to combine data. In the future, changes in the properties of the simulation based on the analysis of this set. It was obtained forecast values relative subsidence, strength, modulus of deformation in natural and humid condition on the basis of the application of inductive methods of research. Quantitative analysis of the results indicates a significant difference between the predicted values of indicators obtained by the regulatory procedure, it points to the possible negative effects of the regulatory processes of forecasting techniques. In the future, we recommend the use of neural network technology to forecasting properties of soils in the area with affected structures.

Key words: Neural network, Physical and mechanical properties of soils, geological forecasting, stochastic simulation, calculation of rainfall, the calculation of drawdown

Введение. Прогноз изменений состояния и свойств грунтов в зоне влияния проектируемых сооружений представляет собой сложную комплексную проблему, решение которой в условиях роста интенсивности техногенных воздействий, возрастания плотности застройки, ухудшения состояния геологической среды требует нового подхода. Стратегия проектирования сооружений основана на использовании принципов максимальной надежности и оправданного риска. При этом в обеих стратегиях вопросам оценки риска, 
возникающего из-за упрощенного подхода к прогнозу процессов, моделированию среды, уделяется недостаточное внимание.

Просадочные лессовые отложения относятся к грунтовым средам, отличающимся особенным поведением в условиях техногенеза, изучение процессов изменений состояния и свойств является предметом исследований ученых России (Romanova, 2009), Украины (Mokritskaja, 2013), Канады (Briaud). Выполняется поиск теоретических моделей, точнее описывающих деградацию структуры этих отложений в процессе деформирования (Mokritskaja, Shestopalov, Tushev, 2012). Экспериментальные исследования направлены на феноменологическое описание текстурно-структурных особенностей грунта, развиваются методы математического моделирования пространственной изменчивости свойств (Dean, 2015; Chaturvedil, Prasad, 2013; Hanaor, Gan, Einav, 2013; Schoenball, Selzer, Kühnle1 and all, 2013). Примеры применения методов индуктивного моделирования (метода группового учета аргументов, метода нейронных сетей) к решению практических задач проектирования и инженерно-геологического прогнозирования немногочисленны, несмотря на широкие возможности. В данной работе рассмотрены некоторые возможности использования указанных технологий для описания изменчивости свойств и прогноза их поведения.

Методы и материалы исследований. В данной работе использованы материалы инженерно-геологических исследований, выполненных на территории, примыкающей к зоне формирования катастрофического оползневого явления суффозионно-просадочного типа (1996 г., г. Днепропетровск). Привлечены материалы изысканий, выполненных для проектирования двух жилых домовв границах одного геоморфологического элемента в 1989 г. и 1997 г. Проектируемые сооружения представляют собой многоэтажные жилые здания, конструктивные особенности и проектируемые нагрузки сооружений близкие.

В геологическом строении площадок принимают участие отложения плейстоценового возраста, изученные на глубину до 26 м. В 1989 году уровень грунтовых вод находился на глубине 4,9 м, а в 1997-м - на глубине 8,2 м. Уровень грунтовых вод опустился на 3,3 м, что связано с влиянием катастрофического оползневого явления (1996 г.). В нормах, регламентирующих методику повторного использования данных, отсутствуют количественные критерии оценки допустимости привлечения данных о свойствах грунтов, что делает возможным привлечение материалов изысканий прошлых лет в полном объеме. Статистический анализ в подобных случаях приводит к ложным выводам. В данной работе анализ изменчивости и прогноз выполнялись методами стохастического и индуктивного моделирования с помощью trial-версии программы STATISTICA 13.0.

Результаты исследований и их обсуждение. Результаты лабораторных исследований свойств грунтов были сведены в единую базу данных, так как изучить изменчивость свойств во времени по результатам двух наблюдений не представляется возможным. Общее количество записей (строк) - 90, приведены данные, полученные на соседствующих площадках, до и после катастрофического события.

Выполненные традиционными методами статистический, корреляционный и регрессионный анализ показали, что полученные модели не могут быть использованы для прогноза, так как значения коэффициента детерминации малы, нормальный закон распределения не подтвержден. Необходим поиск иного метода. Достоверно прогноз изменения деформационных, прочностных показателей в условиях неполноты информации может быть выполнен с помощью индуктивного моделирования, а именно с привлечением нейронных сетей (Stepashko, Bulgakova, Zosimov, 2010; Borovikov, 2008; Rumelhar, Hinton, Williams, 1986). В Украине ранее моделирование изменчивости свойств грунтов с привлечением нейронных сетей не выполнялось.

Выполнено моделирование зависимости показателей просадочности, деформируемости и прочности грунтов от пространственных координат (глубины) и показателей физического состояния. В состав входных переменных были включены: глубина отбора $z$, м, пределы пластичности $\omega_{L}$, $\omega_{p}$, д. ед., плотность грунта $\rho$ и плотность частиц $\rho_{S}, \Gamma / \mathrm{cm}^{3}$. В качестве выходных переменных были выбраны показатели, используемые в задачах проектирования: модуль деформациив состоянии природной влажности и полного водонасыщения $E$ и $E_{s a t}$, МПа, удельное сцепление $c$, МПа и, угол внутреннего трения $\varphi$,град; начальное просадочное давление $P_{s L}$, КПа и относительная просадочность $\sigma_{s L}$, д.ед. С помощью подпрограммы Automated Neural Networks (SANN) решалась задача регрессионного анализа. В качестве целевой функции последовательно выбирали значения показателей физико-механических свойств, при неизменном и описанном выше наборе входных переменных. Во всех случаях использовали стратегию «Автоматизированная нейронная сеть (АНС)», которая требует минимального участия пользователя. Выбирали тип сети - многослойный персептрон, выполняли 
Сравнительный анализ нормативных и прогнозных значений показателей свойств грунтов

\begin{tabular}{|l|c|c|c|}
\hline \multicolumn{1}{|c|}{ Показатель } & $\begin{array}{c}\text { Нормативное } \\
\text { значение }\end{array}$ & $\begin{array}{c}\text { Значение } \\
\text { пользователя }\end{array}$ & Прогнозное значение \\
\hline Модуль деформации грунта естественной влажности, МПа & 14 & 11 & 3 \\
\hline $\begin{array}{l}\text { Модуль деформации грунта в состоянии полного } \\
\text { водонасыщения, МПа }\end{array}$ & 5 & & 0,013 \\
\hline Удельное сцепление, МПа & 0,011 & 0,016 & 24 \\
\hline Угол внутреннего трения, град & 24 & 19 & 0,$003 ;$ \\
\hline Относительная просадочность, д. ед., при давлении 0,05 МПа & 0,005 & - & 0,$011 ;$ \\
\hline Относительная просадочность, д. ед., при давлении 0,1МПа & 0,01 & - & 0,$011 ;$ \\
\hline Относительная просадочность, д. ед., при давлении 0,15 МПа & 0,027 & - & 0,$023 ;$ \\
\hline Относительная просадочность, д. ед., при давлении 0,2 МПа & 0,04 & - & 0,$07 ;$ \\
\hline Относительная просадочность, д. ед., при давлении 0,25 МПа & 0,057 & - & 0,062 \\
\hline Относительная просадочность, д. ед., при давлении 0,3 МПа & 0,065 & - & \\
\hline
\end{tabular}

обучение пяти сетей на пяти подвыборках. Анализ гистограмм остатков для каждой из сетей, диаграмм рассеяния, поверхностей отклика выполнялся совместно. Также выполнялся анализ глобальной и локальной чувствительности сети, анализ качества сети в соответствии с методикой, описанной разработчиками программы (Bishop, 1995).

Значения входных переменных для прогноза модуля деформации $E$, МПа, задавали, предполагая, что изменение состояния грунта по влажности отразится на значениях показателей пластичности и плотности, но не отразится на значениях плотности частиц. Интервал возможных изменений влажности определяли величиной интервала от природной влажности до влажности на границе текучести. Деление интервала на три части позволяет выделить три области состояния с разной интенсивностью изменений свойств. В качестве входных задавались переменные, находящиеся в границах указанных областей. Для прогноза уплотнения выбирали прогнозное значение модуля деформации, полученное с наименьшей ошибкой. Результаты расчетов приведены ниже (табл. 1).

\section{Результаты прогноза осадки и просадки}

\begin{tabular}{|c|c|c|c|c|c|c|}
\hline \multirow{2}{*}{ Объект } & \multicolumn{4}{|c|}{ Осадка, м } & \multicolumn{3}{c|}{ Просадка, м } \\
\cline { 2 - 7 } & \multicolumn{2}{|c|}{ Вариант прогноза 1 } & \multicolumn{2}{|c|}{ Вариант прогноза 2 } \\
\cline { 2 - 7 } & $\begin{array}{c}\text { Нормативная } \\
\text { методика }\end{array}$ & $\begin{array}{c}\text { Индуктивное } \\
\text { моделирование }\end{array}$ & $\begin{array}{c}\text { Нормативная } \\
\text { методика }\end{array}$ & $\begin{array}{c}\text { Индуктивное } \\
\text { моделирование }\end{array}$ & $\begin{array}{c}\text { Нормативная } \\
\text { методика }\end{array}$ & $\begin{array}{c}\text { Индуктивное } \\
\text { моделирование }\end{array}$ \\
\hline 1 & 0,035 & 0,055 & - & 0,129 & 0,289 & 0,163 \\
\hline 2 & 0,024 & 0,036 & - & 0,015 & - & - \\
\hline
\end{tabular}

В дальнейшем были выполнены расчеты величин осадок и просадки в основании проектируемых сооружений на примере двух соседних площадок. Расчетная схема грунтовых оснований площадок 1 и 2 приведена на рисунках 1 и 2.
При расчете деформаций оснований зданий (объекты 1 и 2) последовательно привлекались нормативные и прогнозные значения показателей свойств грунтов для количественной оценки величины деформаций в двух вариантах. Первый вариант расчета осадки определен условиями, соответствующими состоянию на момент завершения строительных работ, на объекте 1 грунты находились в состоянии природной влажности, на второй площадке - в состоянии естественной влажности. Второй вариант расчета учитываетизменение состояния по влажности грунтов в зоне аэрации. На объекте 1 моделируется переход в состояние полного водонасыщения, а на объекте 2 - снижение влажности и повышение значений модуля деформации. Нормативные значения показателей свойств грунтов рассчитывались по действующей в инженерной геологииметодике статистической обработки данных. Прогнозные значения рассчитывались по результатам индуктивного моделирования с привлечением подпрограммы Automated Neural Networks (SANN). Просадка основания возможна на объекте 1 , вели-

Таблииа 2 чина просадки за счет дополнительного давления выполнена по стандартной методике. Результаты прогноза деформаций просадки и осадки приведены в таблице 2. 


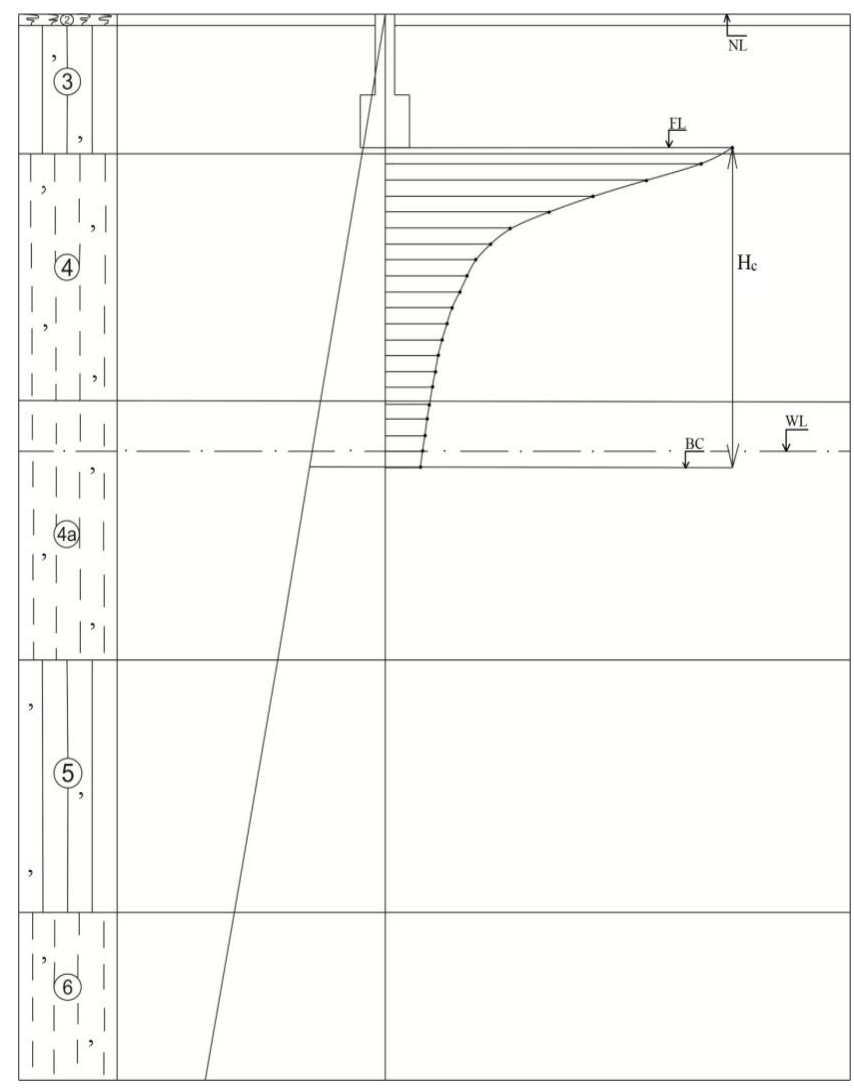

Рис. 1. Расчетная схема к определению осадки основания

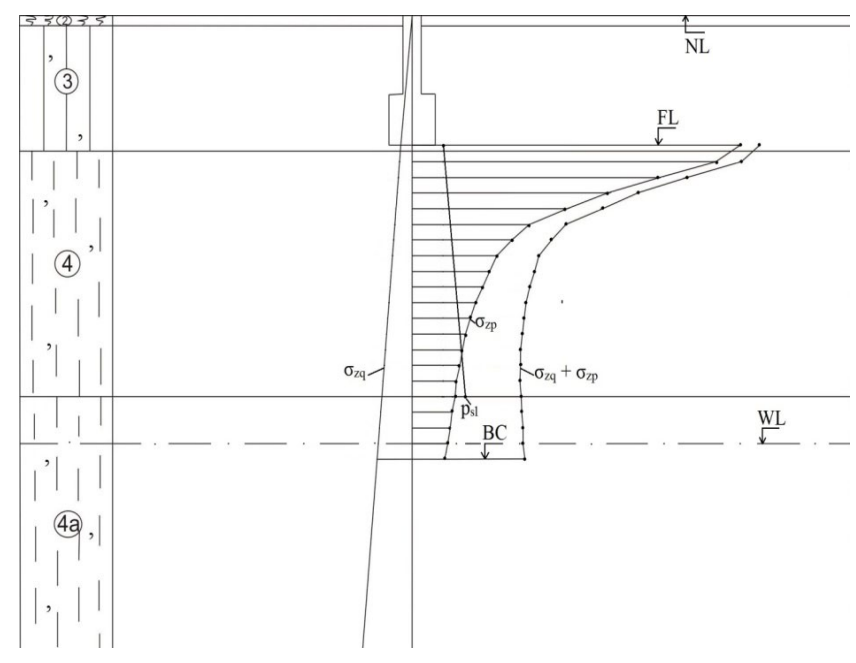

Рис. 2. Расчетная схема к определению просадки основания

Условные обозначения для рисунка 1 и 2:

2, 3, 4, 4a, 5, 6 - номер инженерно-геологического элемента,

$N L$ - отметка поверхности естественного рельефа,

$F L$ - отметка подошвы фундамента,

$W L$ - уровень подземных вод,

$B C-$ нижняя граница сжимаемой толщи.
Анализ результатов показывает, что применение индуктивного моделирования позволяет выполнить моделирование различных состояний грунтов основания, получить более реалистичные оценки показателей физико-механических свойств.

\section{Выводы}

- Использование материалов изысканий прошлых лет при проектировании сооружений должно быть строго обосновано.

- Использование методов индуктивного моделирования позволяет получить более реалистичные оценки показателей физико-механических свойств грунтов, учитывающие изменение их состояния.

\section{Библиографические ссылки}

Bishop, C., 1995. Neural Networks for Pattern Recognition. Clarendon Press, Oxford.

Borovikov, V. P., and all, 2008. Nejronnye seti. STATISTICA Neural Networks: Metodologija i tehnologii sovremennogo analiza dannyh.[Neural networks. STATISTICA Neural Networks: Methodology and tehnology of modern data analysis] M.: Hot line, Telekom. (in Russian)

Briaud, J.L. 2013. Introduction to geotechnical engineering : unsaturated and saturated soils. Wiley. $1022 \mathrm{p}$.

Chaturvedil, A., Prasad P. R. Ch., 2013.Application of fractal geometry in determining optimal quadrat size for vegetation sampling. Current Science, vol. 105, 9, 1275-1281. cite web |url=http://www.statsoft.ru/home/textbook/default. htm StatSoft. Jelektronnyj uchebnik po statistike.

Dean, E. T. R., 2015. Particle mechanics approach to continuum constitutive modelling. Geotechnical Research, 2(1), 3-34.

Hanaor, D.A.H., Gan, Y. Einav, I., 2013. Effects of surface structure deformation on static friction at fractal interfaces. Geotechnique Letters 3, № 2, 52-58. doi.org/10.1680/ geolett.13.016

Mokritskaja, T.P., 2013. Peculiarities of degradation in loess soils' deformation and strength properties on the example of Dnipropetrovsk city. Visnyk KNU im. T.Shevchenka, serija Geologija, 62 (3), $66-70$.

Mokritskaja, T.P., Shestopalov, V.M., Tushev, A.V., 2012. Some facts on relations between stability and fractality of geological structures. Fundamental and Applied Science Problems, Vol 4, M: RAN, 8-16. (in Russian)

Romanova, M. V., 2009. Kompleksnyj podhod k racional'nomu ispol'zovaniju zemel'nyh resursov pri stroitel'nom osvoenii territorij na osnove ocenki geotehnicheskogo riska. [An integrated approach to the rational use of land recources in the construction development of the territory based on the geotechnical risk assessment] Bulletin ChitGU, $1(52), 100-105$.

Rumelhart, D.E., Hinton, G.E., Williams, R.J., 1986. Learning representations of back-propagation errors. Nature, 323, $533-536$. 
Schoenball, M., Selzer, M., Kühnlel, N., Nestler, B., Schmittbuhl, J., Kohl, T., 2013. Flow anisotropy in sheared fractures with self-affine surfaces. European Geothermal Congress, Pisa, Italy.

Stepashko, V.S., Bulgakova, O.S., Zosimov, V.V., 2010.

Поступила в редколлегию 25.01.2016
Gibrydni algorytmy samoorganizacii’ modelej dlja prognozuvannja skladnyh procesiv. [Hybrid algorithm self-models to predict complex processes] The induktive modeling of complex systems. Proceedings, is. 2, Kyiv: MNNC ITS, 236 - 246. 